\section{ULTRADEX PROTECTS, RESTORES AND ELIMINATES}

Preventive care incorporating exceptional plaque control and the elimination of volatile sulphur compounds, together with tissue maintenance, is of critical importance for patients at risk of periodontal problems, often linked to bad breath and other oral hygiene issues. Working closely with the natural oral $\mathrm{pH}$, the UltraDEX Performance Oral Care range can be a significant aid in the fight against bad breath, tooth decay, plaque and gum problems, when used as part of an oral hygiene regime.

The clinically proven $\mathrm{iQ+}$ Active0xi
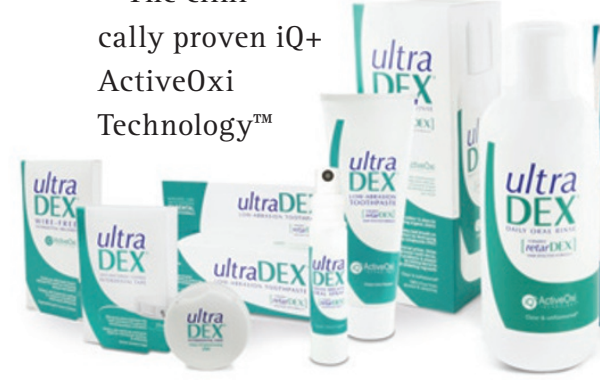

with stabilised chlorine dioxide helps to prevent the formation of plaque biofilm, inhibits its regrowth and instantly eliminates volatile sulphur compounds for at least 12 hours of fresh breath. Patient compliance is excellent: there are no unpleasant side-effects such as staining or interference with taste sensation; instead the technology gently lifts off organic stains by a process of oxidation.

Safe for long-term daily use the range bridges the gap between professional and at 'home care'. UltraDEX products contain fluoride; are free from alcohol, colouring and sodium lauryl sulphate; are approved by the Vegetarian Society; are registered with the Vegan Society; and are gluten free. Personalised UltraDEX Oral Care Kits are available to purchase directly from Perioproducts and are a great tool to increase practice profile and ensure the products ultra
DEX DEX ultra ultra For further information please DEX ultraDEX visit www.periproducts.co.uk or email 1. ultraDEX one of the team at dental@periproducts.co.uk.

\title{
INTEGRATE YOUR CLINICAL IMAGING SYSTEM
}

Dentally has recently announced integrations with even more clinical imaging systems in the practice. Dentally supports all image types, directly in the cloud, from radiographs to intraoral and extraoral photographs.

Integrations have already been released to facilitate imaging with DBSWIN from Duerr, Trophy / Kodak / Carestream and VixWin from Gendex, with many others on the way. This allows the practice to choose from a wide range of sensors, phosphor plate systems or intraoral camera setups.

Exciting developments are also on the horizon to provide seamless and secure upload of clinical images to the cloud additionally via DentalEye from Vatech and VistaScan for Mac from Duerr. Images will be readily accessible from anywhere the practitioner needs to get their work done, including viewing both X-rays and photos on an iPad

Dentally is beautiful and functional software that sits at the centre of your practice, allowing you to run your business from anywhere. Read more about our sophisticated imaging in the tour section at www.dentally.co/bdj.

\section{PUTTING INNOVATION INTO PRACTICE}

The world of dentistry is evolving and it is vital to stay innovative and up to date with modern practices.

BDIA Dental Showcase offers you the opportunity to speak with the leading companies and suppliers who are at the forefront of the dental industry. They will also be offering over 50 short, focused lectures on their products and services, all qualifying for verifiable CPD.

BDIA Dental Showcase takes place at NEC Birmingham from 22-24 0ctober 2015. Save the dates now! For further information on the UK's biggest dental exhibition, visit www.dentalshowcase.com.

\section{D IMAGING: ACCESSIBLE TO ALL}

The Carestream CS8100 3D, available from Henry Schein Dental, effectively blends award-winning and cutting-edge panoramic 2D imaging with 3D capability, in a lightweight and ultra-compact design.

Recommended for general practitioners and specialists alike, the CS8100 3D meets the varying demands of daily practice offering a choice of 2D mode to conduct traditional panoramic exams and 3D mode for more advanced applications such as endodontics, implant planning and oral surgery.

The unit's intuitive interface and easy-to-use imaging software is supported by preset programmes and automatic exposure settings, making examinations faster and easier, and helping to eliminate operational errors. Utilising cone beam computed tomography (CBCT) technology, the unit produces a significantly lower radiation dose than the average CBCT system and offers four selectable fields of view and a fast scanning mode, collimating radiation to the area of interest and reducing the length of exposure.

Easier positioning, faster image acquisition, and higher image quality means the CS8100 3D can give you everything you need to streamline your workflow and make more accurate, real-time diagnoses that improve patient care.

For more details contact your local Henry Schein Digital Specialist on 08700102041 or visit www.henryschein.co.uk. 